\title{
Visualizing the Entry of Clostridium difficile Toxin A into Human Colonic Epithelial Cells
}

\author{
Ramyavardhanee Chandrasekaran ${ }^{1}$ and D. Borden Lacy ${ }^{1,2}$ \\ ${ }^{1 .}$ Department of Pathology, Microbiology and Immunology, Vanderbilt University School of Medicine, \\ Nashville, TN 37232, USA. \\ 2. The Veterans Affairs Tennessee Valley Healthcare System, Nashville, TN 37212, USA.
}

Clostridium difficile is a gram-positive, spore-forming anaerobe that infects the human colon following antibiotic-induced changes to the gut microbiota. Symptoms of $C$. difficile infection (CDI) typically range from uncomplicated diarrhea to life-threatening colitis [1]. CDI is currently the leading cause of antibiotic-associated intestinal disease in the U.S., with health care costs exceeding $\$ 3$ billion per year $[2,3]$. Outbreaks of multi-drug resistant and hypervirulent strains have led to treatment failures, higher incidence of recurrence and increased mortality rates, creating an urgent need for new therapeutic strategies to treat CDI [4].

The pathogenesis of $C$. difficile is mediated by two large homologous exotoxins, Toxin A (TcdA; 307 $\mathrm{kDa})$ and Toxin $\mathrm{B}(\mathrm{TcdB} ; 270 \mathrm{kDa})$. The toxins are glucosyltransferases that inactivate Rho familyGTPases such as RhoA, Rac1 and Cdc42. Inactivation of these GTPases results in a loss of actin cytoskeletal architecture of cells leading to cell rounding (Fig. 1A and B) and cell death [1]. Cellular toxicity mediated by $C$. difficile toxins therefore depends on receptor binding and subsequent endocytosis into cells. Since these toxins represent excellent targets for therapeutic intervention, understanding the mechanism of toxin entry is a significant priority.

Mammalian cells have several endocytic pathways to internalize nutrients, cargo proteins and to recycle cell surface receptors [5]. These endogenous cellular mechanisms are often exploited by pathogens and virulence factors that hijack the cellular machinery to promote entry into target cells. In the case of $C$. difficile toxins, TcdB has been shown to utilize clathrin-dependent endocytosis to induce cell rounding [6] but the mechanism of TcdA uptake is still unclear. Previous investigations on TcdA uptake implicate both clathrin- and dynamin-dependent and independent pathways, and have relied primarily on inhibitor studies and indirect entry read-outs such as GTPase inactivation and cell rounding [6, 7]. In order to conclusively identify the endocytic pathway(s) exploited by TcdA for intoxication, it was important that we establish an imaging-based system to directly visualize toxin entry in cells. We tried three different strategies for visualizing toxin entry by confocal microscopy. The first strategy involved generating $\mathrm{N}-$ terminal AviTagged-TcdA by site-directed mutagenesis, followed by enzymatic biotinylation with an $E$. coli biotin ligase (BirA). The biotinylated toxin was mixed with a ten-fold molar excess of streptavidinquantum dot (Qdot) conjugates, added to cells, and imaged with an Olympus FV-1000 confocal microscope. Qdot-conjugation allowed us to visualize lower nanomolar quantities of toxin within cells (Fig. 1C and D). However, we did not proceed with this approach due to concerns that the qdots might be altering the entry of toxin, as was previously reported for transferrin and shiga toxin [8]. Our second strategy involved addition of a 3xFLAG tag at the N-terminus of TcdA by site-directed mutagenesis and using anti-FLAG antibodies to detect toxin entry in cells. Our signal-to-noise ratio with this strategy was poor and was not sufficient to perform colocalization studies. Our third strategy involved directly labeling TcdA with reactive Alexa dyes. We were able to label TcdA with thiol-reactive Alexa546, with no loss in toxin function. Caco-2 (human colon adenocarcinoma) cells intoxicated with TcdA-546 were 
fixed, stained for clathrin and imaged using a 63x objective (LSM 710 Meta Inverted Confocal Microscope). We did not observe any colocalization of TcdA with clathrin-positive vesicles (Fig. 1D and E) suggesting that TcdA and TcdB might be exploiting different entry pathways to intoxicate cells. Using these labeled toxins as tools, we are now investigating the endogenous endocytic mechanisms of host cells that are hijacked by TcdA to enter and intoxicate their target cells.

References.

[1] Voth, D. E. and J. D. Ballard, Clin Microbiol Rev 18 (2005), p. 247-63.

[2] Dubberke, E. R. and M. A. Olsen, Clin Infect Dis 55 Suppl (2015), p. S88-92.

[3] Dubberke, E. R. and A. I. Wertheimer, Infect Control Hosp Epidemiol 30 (2009), p. 57-66.

[4] Kelly, C. P. and J. T. LaMont, N Engl J Med 359 (2008), p. 1932-40.

[5] Doherty, G. J. and H. T. McMahon, Annu Rev Biochem 78 (2009), p. 857-902.

[6] Papatheodorou, P., et al., PLoS One 5 (2010), p. e10673.

[7] Gerhard, R., at al., J Med Microbiol 62 (2013), p. 1414-22.

[8] Tekle, C., et al., Nano Lett 8 (2008), p. 1858-65.
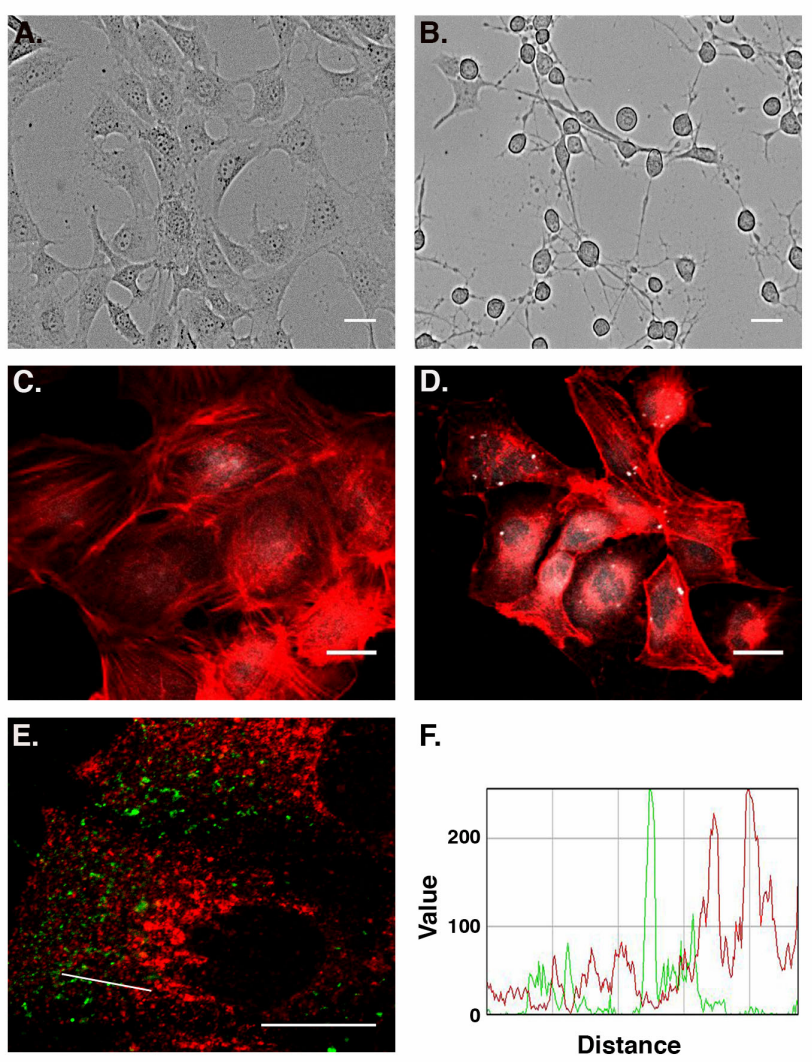

F.

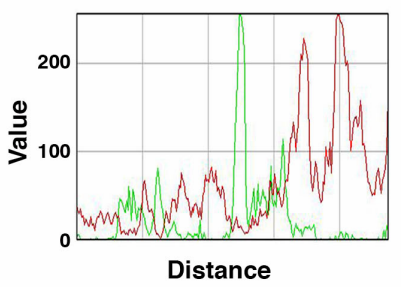

Figure 1. Fluorescently labeled toxin as a tool to visualize and identify TcdA entry mechanism(s) in cells. Cells before (A) and after addition (B) of $10 \mathrm{nM}$ TcdA show the cytopathic 'cell rounding' effects induced by toxin. Images were obtained using a Cytation 5 multi-mode plate reader. Scale bar, 100 pixels.

Colonic epithelial cells were treated with Qdot605 only (C) or $1 \mathrm{nM}$ of TcdA-Qdot605 (D; gray puncta), fixed and stained for actin (red). Toxintreated cells show signs of rounding indicating that the Qdot-conjugation does not affect the function of toxin. Images were obtained using a $60 \mathrm{x} / 1.45$ Plan-Apochromat oil lens (Olympus FV-1000). Scale bar, $20 \mu \mathrm{m}$.

E) Colonic epithelial cells treated with $50 \mathrm{nM}$ TcdA-546 (green) for $20 \mathrm{~min}$ at $37{ }^{\circ} \mathrm{C}$, fixed and stained for clathrin (red). Images were obtained using a $63 \mathrm{x} / 1.40$ Plan-Apochromat oil lens (Zeiss LSM 710 Meta Inverted). Scale bar, $20 \mu \mathrm{m}$. F) RGB profile analysis of the tracing shown in (E) reveals a lack of colocalization between TcdA and clathrin. 